\title{
Distributed Design Review in Virtual Environments
}

\author{
Mike Daily Mike Howard Jason Jerald Craig Lee Kevin Martin Doug Mclnnes Pete Tinker \\ HRL Laboratories \\ 3011 Malibu Canyon Road \\ Malibu, CA 90265 USA \\ +13103175673 \\ mjdaily@hrl.com \\ Randall C. Smith \\ GM Research and Development Center \\ 30500 Mound Road, Box 9055 \\ Warren, Michigan 48090-9055 \\ $+18109861461$ \\ smith@gmr.com
}

\begin{abstract}
In large distributed corporations, distributed design review offers the potential for cost savings, reduced time to market, and improved efficiency. It also has the potential to improve the design process by enabling wider expertise to be incorporated in design reviews. This paper describes the integration of several components to enable distributed virtual design review in mixed multi-party, heterogeneous multi-site 2D and immersive 3D environments. The system provides higher layers of support for collaboration including avatars, high fidelity audio, and shared artifact manipulation. The system functions across several interface environments ranging from CAVEs to Walls to desktop workstations. At the center of the software architecture is the Human Integrating Virtual Environment (HIVE) [6], a collaboration infrastructure and toolset to support research and development of multi-user, geographically distributed, 2D and 3D shared applications. The HIVE functions with VisualEyes software for visualizing 3D data in virtual environments. We also describe in detail the configuration and lessons learned in a two site, heterogeneous multi-user demonstration of the system between HRL Laboratories in Malibu, California and GM R\&D in Warren, Michigan.
\end{abstract}

\section{Keywords}

Virtual environments, multi-modal, global scale collaboration, design review, tele-conferencing, spatialized audio, speech recognition

(C) 2000 HRL Laboratories, LLC. All Rights Reserved

\section{INTRODUCTION}

Visualization and Virtual Environments are critical enablers for next generation 3D model-based commercial design and development processes. Replacing physical mockups for analysis of design aspects with virtual models saves cost and reduces time to market. Geographically distributed users need a common context, visualization, and natural communication for design review. Worldwide-distributed design review offers the potential for increased cost savings, reduced time to market, and improved efficiency. It also has the potential to improve the design process by enabling wider expertise to be incorporated in design reviews, reducing the need for travel, and further reducing dependence on physical models.

This paper describes the integration and application of capabilities developed at HRL Laboratories and General Motors Research \& Development Center (GM R\&D) in a system for Distributed Design Review In Virtual Environments (DDRIVE) (Figure 1). A central goal of the effort was to extend the single site functionality of GMR\&D's visualization software by constructing a prototype collaboration environment to match the performance of the available network and computing infrastructure. Collaboration infrastructure development to support seamless integration of a range of collaboration applications was emphasized. The two main software features of the DDRIVE system are:

- The Human Integrating Virtual Environment (HIVE) collaboration infrastructure and toolset developed at HRL Laboratories to support research in multi-user, geographically distributed, 2D and 3D shared applications. It uses VRML 2.0 for scene graph descriptions, Java or $\mathrm{C} / \mathrm{C}++$ for application development, and Voyager [3] for communication and synchronization. The HIVE provides virtual meeting tools and high-fidelity multi-way audio communication for heterogeneous interface environments. The HIVE is designed to work cooperatively with other applications, to add collaboration capabilities. 


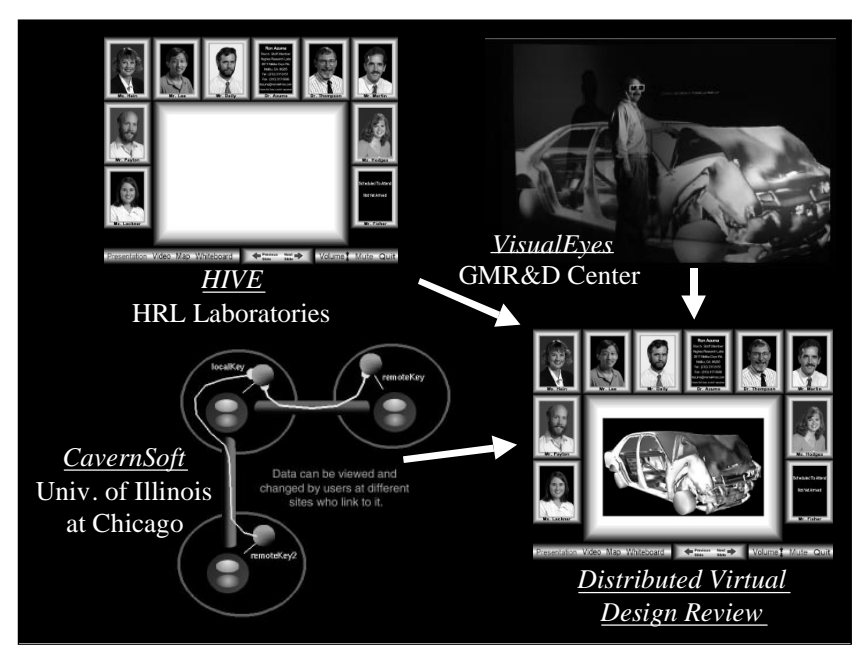

Figure 1. Components of the DDRIVE system.

- VisualEyes [17] provides a full size immersive view of a shared model for high quality visualization. VisualEyes is an interaction tool for visualizing data using mathematics and light. GM uses this tool for testing car designs and collaborating on projects in virtual environments. Imagine being able to sit in the driver's seat of a car before anything is built and change the placing of a dashboard control by a simple gesture because it's too difficult to reach. VisualEyes enables this kind of interactive design via an easy-to-use scripting language that allows control of the environment. Virtual environments can be built much more quickly than with other toolkits by merely bringing in models and applying simple rules.

A unique aspect of the DDRIVE system is the support for heterogeneous interface and hardware consisting of the following:

- The HRL CABANA [2]: a re-configurable large format spatially immersive display in Malibu, California.

- The GM R\&D CAVE and PowerWall: a four-wall CAVE and two screen PowerWall in Warren, MI.

- Desktop workstations: multiple SGI workstations running the HIVE and VisualEyes at both locations, PC Windows workstations for speech recognition.

- Dedicated ISDN: used for communication between Malibu and Warren.

We describe performance issues and lessons learned in the final sections.

\section{PREVIOUS COLLABORATIVE WORK}

Collaborative Virtual Reality requires the consideration of how participants should interact with each other in a shared virtual space while being physically separated by great distances. Recent advances have focused on recreating a remote sense of presence in addition to immersive interaction with 3D data. For a good review of recent CAVE related efforts see [8].

In 1997 HRL Laboratories built one of the first three-way geographically dispersed shared virtual environments for training of space station personnel for Hughes Training Inc. and NASA [10]. This system provided users at three remote sites the ability to move and collaborate in a shared model of the international space station.

A key component to effective collaborative virtual environments is the communication infrastructure. CAVERNSoft [9] from UIC EVL is a software library that allows VR developers to share information between their applications. This is a hybrid system that combines a distributed shared memory model with distributed database technology and real-time networking technology. This allows objects manipulated by one user to immediately be perceived by all other users at remote locations. The VisualEyes software uses this library to communicate keys or messages among components.

Collaborative VisualEyes [16] is a retrofitted version of VisualEyes enabling global scale collaboration between VisualEyes applications. Collaborative VisualEyes clients share 3D scene graph information by directly linking individual data nodes over a communication channel implemented with CAVERNSoft.

Related work in desktop collaboration includes Habanero [4], NetMeeting [12], Placeware [15], and the MASH toolkit [11]. These are products for connecting participants with roughly equivalent hardware capabilities into a conference session using a 2D Graphical User Interface (GUI). Microsoft NetMeeting, the most widely used, is limited to toll-quality audio. The MASH toolkit is actually a set of modular programs, including audio and video feeds and a whiteboard, all controlled by a session manager. A number of types of encoding are available, and higher quality audio is available (though in our experience the repair of lost packets is not very good). Placeware is a Java collaboration toolkit that provides the metaphor of "rooms". A user sees what is going on in a room, and upon entry, can communicate with other users in the room. There is a text chat facility, but no audio.

\section{SYSTEM ARCHITECTURE}

Collaborative virtual environments require all of the system level functionality of single site, singer user virtual environments to recreate immersive data. In addition, CVEs require networking infrastructure to support communication and synchronization between application software at each site. In this section, we first describe the hardware environment of both sites and the network infrastructure to support distributed design review. Next, key software modules that execute at both sites is explained. Speech and audio is then covered.

\subsection{Hardware}

The CAVE [1] (CAVE Automatic Virtual Environment) was developed at the Electronic Visualization Laboratory (EVL) at University of Illinois Chicago. In the DDRIVE system, several immersive display systems have been used for the development, experimentation, and demonstration. These include a standard CAVE located at GM R\&D, A PowerWall located at the same site, and the CABANA (CAVE At the Beach And Nearby Attractions) designed at $\mathrm{HRL}$ as a re-configurable immersive display unit (Figure 2). The walls of the CABANA can be 


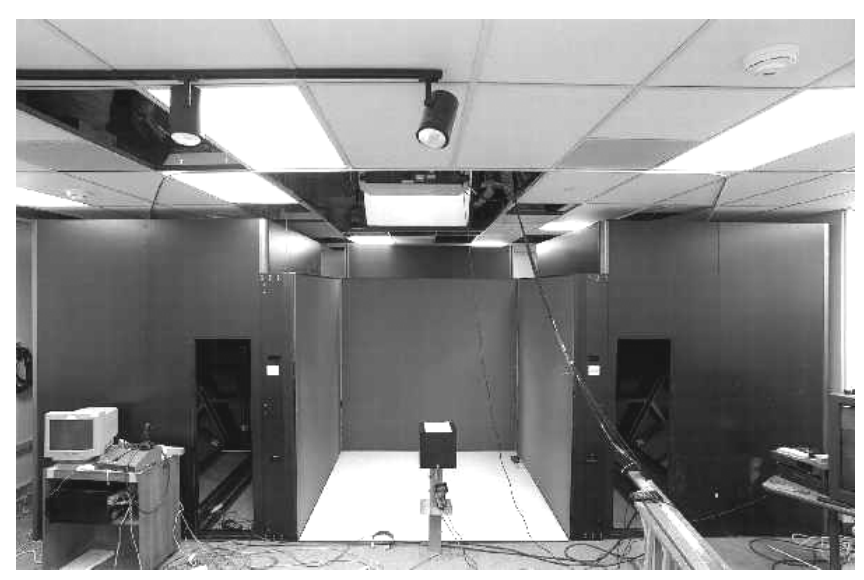

Figure 2. The CABANA in CAVE mode.

physically transformed to suit the needs of the application. Examples include a CAVE like room and a high-resolution wall.

In addition, the system uses several SGI workstations and PCs. The SGI workstations provide non-immersive views of the collaboration session along with audio interface into the session. The PCs are used as speech recognition and audio engines, sending recognized commands to the VisualEyes software and playing audio files on command.

To support the requirement of distributed design review, we determined that a dedicated network was essential in order to reduce latency and variability of latency in delivery of packets between sites. Since the DDRIVE system only requires state information and higher quality audio be transported between sites, we chose to use a low cost dedicated ISDN line for the communications infrastructure in the final demonstration system. The limited bandwidth of ISDN made it essential to use bandwidth efficiently.

\subsection{Software}

The HIVE provides an extensible infrastructure for collaboration. The number and variety of projects using the HIVE mandate a diverse set of requirements:

- It supports connecting an unrestricted number of participants at different host machines into a collaboration session.

- It supports client hardware with varying abilities to render graphical and audio data.

- It integrates shared applications (i.e., applications that exchange data among themselves) into the collaboration session.

- It integrates a high-fidelity audio module in the environment and transmits speech between conference participants.

The goal of the HIVE is to support so called "poly-modal" collaboration among participants with arbitrary hardware and software suites. For example, a person using a Personal Digital Assistant (PDA) could collaborate with someone using a traditional 2D-windowing workstation display, and with someone else using an immersive 3D display. Each views the same collaboration session in a form appropriate to their capabilities.

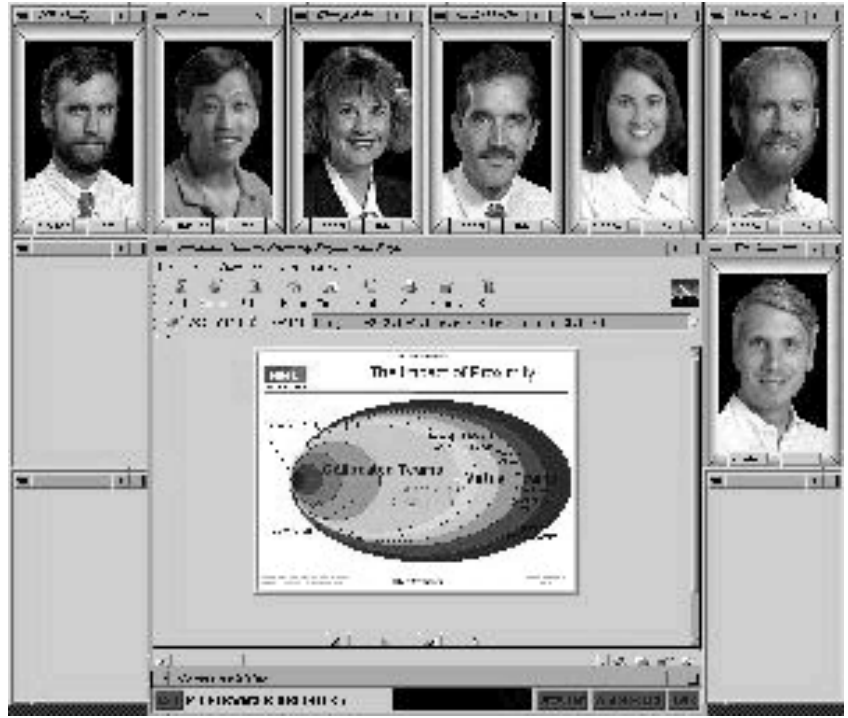

Figure 3. The Hive standard configuration.

In a 2D window-based conferencing application, the HIVE presents images of each participant in the conference, a shared whiteboard, and a shared browser that can be used to review viewgraphs or other data (Figure 3). The HIVE incorporates a variety of techniques such as speaker highlighting to provide additional non-verbal cues about who is speaking. The highfidelity audio module of the HIVE connects multiple clients together in a multicast group and enables sidebar conversations apart from the main group. Communication between HIVE clients is done using Voyager. CAVERNSoft is used for communication between the HIVE and VisualEyes. CAVERNSoft creates a distributed shared memory for client applications that subscribe to data indexed by "keys."

The HIVE architecture and data structures enable collaboration between 2D and 3D clients simultaneously. Geometric information is shared in the form of scene graphs using VRML 2.0 as the $3 \mathrm{D}$ modeling language for dynamic manipulation of the scene and extension with prototype nodes. 3D clients and "shrink-wrapped applications" can also be added to the 3D scene [7]. This is accomplished by "spoofing" the X server and texturing polygons with periodic snapshots of the application's GUI. VisualEyes is one example of a 3D client for immersive 3D visualization, although we also use other $3 \mathrm{D}$ clients based on WorldToolKit [20] and the CAVE libraries [18]. However, in the DDRIVE experiment, 3D model information was shared using the Collaborative VisualEyes mechanism, rather than the HIVE's VRML capabilities.

The 3D interface can support use of 2D interface elements textured on polygons, or completely different metaphors such as a virtual conference table (Figure 4). The buttons on the polygon with the facial images work similar to the $2 \mathrm{D}$ case.

Figure 5 shows software components and their relationship for an example configuration of the DDRIVE system. 


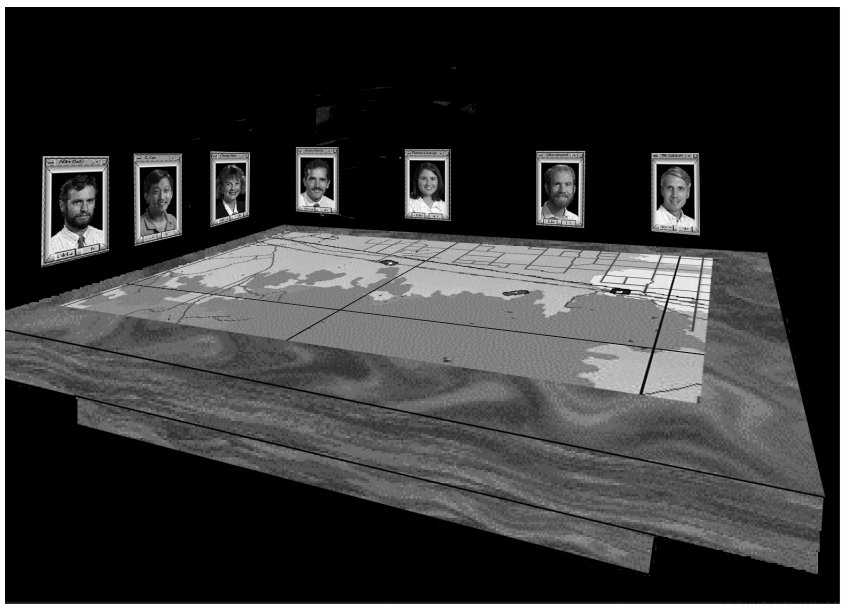

Figure 4. A perspective view rendering of a HIVE collaboration session.

\subsection{Speech and Sound Server}

The HIVE takes advantage of the available hardware and supports a modular upgrade path. This section briefly describes the speech command recognition system and sound server, which enhance the basic functionality of the HIVE. The speech server and the sound server can be distributed on two separate PCs, or coexist on a single PC.

The speech server is designed to support enhanced interaction with the normal command set of VisualEyes, primarily in a single user mode. It uses the spoken language systems toolkit from the Oregon Graduate Institute (OGI)[13].

The OGI Toolkit speech recognition system is called RAD (Rapid Application Developer). One possible way to conceptualize it is as a type of state machine, with matches between spoken words and node specific words/phrases causing transitions to other "state" nodes. Tcl/Tk [14] code may be executed upon entering or exiting a node. Various audio parameters and recognition parameters may be adjusted upon a per node basis or globally

The speech server communicates upon recognition of any of the defined components by sending the recognized word/phrase to a CAVERNSoft key, accessible by VisualEyes.

The sound server acts as an audio gateway between sites that are not connected by a local area network. It collects the multicast audio on the local network, merges it into one stream, optionally compresses it, and sends it to other sites. Site to site transmission is currently accomplished over dedicated ISDN or limited-use T1, but as the wide-area net is upgraded it will become possible to stream higher quality audio over the wide area net.

Spatial audio can improve the effectiveness of a collaborative virtual environment by rendering participant's voices and sounds with spatial context [19]. Due to the recent interest by game designers, sound-spatializing hardware has moved from the research labs to compact chip sets in the past 10 years, and is now available on most audio boards for PCs. The 3D effect is created by mathematically reproducing the frequency transformations that occur when sounds are reflected by the outer ear, head, and upper

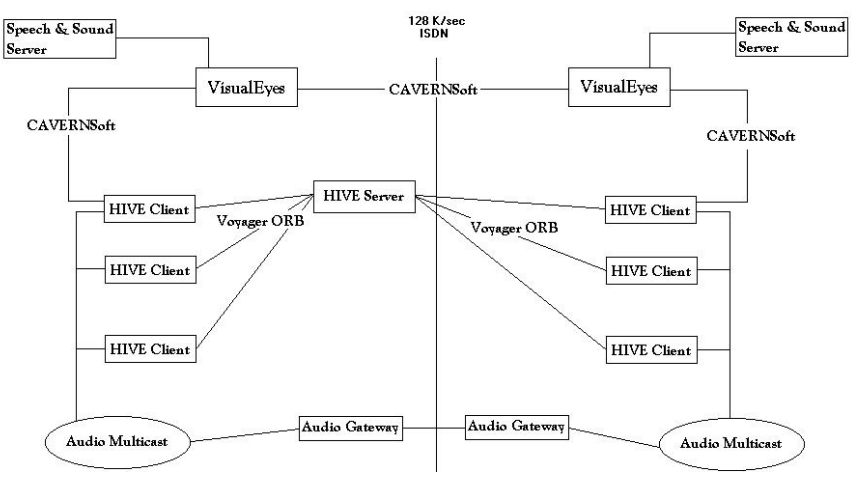

Figure 5. Software components of DDRIVE.

body. The transformation is called a "Head-Related Transfer Function" (HRTF). Spatial audio gives the user a natural cue about the location of people and things in the virtual world. When the user is immersed in a virtual world, playing a sound from a specific location can be the most effective way to alert the user that something is happening there. When multiple people are speaking simultaneously and their speech is rendered spatially, the identification and understanding of a speaker is greatly improved (known as the "cocktail party" effect).

\section{DDRIVE DEMONSTRATIONS}

Several experiments and demonstrations were conducted between remote sites. Several issues were brought to our attention during these sessions that otherwise would not be problematic with a single user environment.

In our first experiment, we conducted a two-site, multiparty pilot demonstration of key components of the DDRIVE system. The configuration employed a hybrid audio system. The demonstration used a combination of VisualEyes over ISDN, the HIVE, POTS telephone, and dedicated networks for audio communication of voice and data. Clients at each geographic site exchanged user-tagged high-quality audio packets, which were mixed for each user in real time. This method delivered hostidentified high-quality audio (16Khz 16 bit samples) for each user. Bandwidth requirements made this technique impractical over long-haul IP networks; our demonstration solution used standard long-distance telephone lines to join the two sites. Each site supported a "virtual client" that translated analog audio from the phone line into digital audio packets on multicast, and visa versa. The result was high-quality speaker-tagged audio among local clients, and telephone quality non-tagged audio to and from remote clients.

In this first study, a number of issues related to the use of mixed analog and digital audio were problematic. Subsequently, we chose to develop the necessary infrastructure to remove the need for the analog phone link, and support digital audio transmission over dedicated ISDN. The final demonstration system possessed the following key capabilities (Figures 6 and 7):

- Integration between HIVE and VisualEyes clients.

- Compressed digital audio via ISDN.

- Avatar and VisualEyes state information via ISDN. 


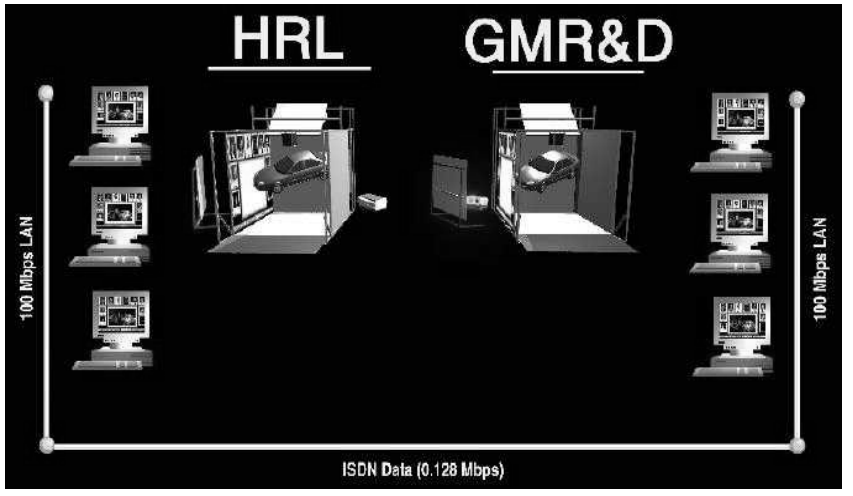

Figure 6. Configuration for DDRIVE demonstration.

- HIVE state information via ISDN

Efforts between the pilot and final demonstrations included new experiments, improvements to the HIVE clients, analysis of bandwidth requirements, and the development of digital audio compression at each audio gateway. To better understand the HIVE audio and VisualEyes bandwidth utilization, we experimented with and optimized the ISDN and local area network infrastructure we used for the inter-site collaboration. Initially our experiments involved two remote local area networks (LANs) tied together by an ISDN based extranet. Each LAN supported fast Ethernet (100Mbps), which we believed would be adequate to support the 3-8 full duplex (i.e. everyone talking simultaneously) high fidelity audio streams and the virtual environment state update information. We collected network traffic data and confirmed that the audio bandwidth utilization was an order of magnitude greater than the environment update bandwidth utilization. The graph in Figure 8 demonstrates the

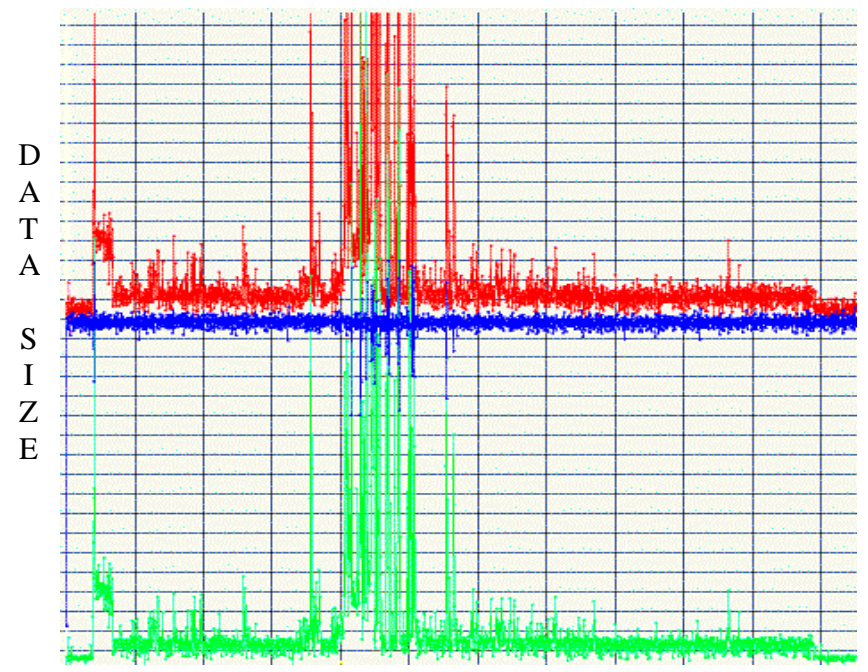

T I M E

Figure 8. Graph showing that virtual environment updates are a minor portion of total packets in the system. Number of

Audio (center band) and VisualEyes (bottom band) packets are graphed against a short time period. Total packets are graphed in the top band.

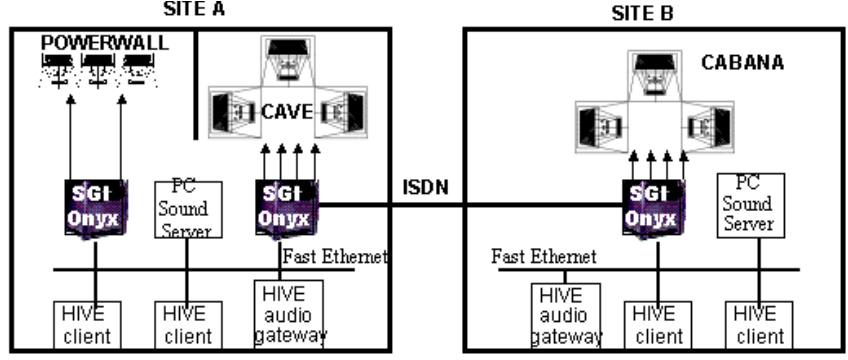

Figure 7. Computing infrastructure for demonstration.

disparity in traffic. The lower band represents reliable TCP/IP based virtual environment state update traffic. The middle band represents heavy utilization of the quick but unreliable User Datagram Packets (UDP) of audio traffic. The high bandwidth in the middle of the $\mathrm{X}$-axis represents $\mathrm{X}$-window based debugging and development traffic. The upper band represents the total of all the traffic between sites.

The inter-site communication via ISDN $(0.128 \mathrm{Mbps})$ severely restricted the transmission of high fidelity audio between sites. As a result, we compressed all of each site's outgoing audio bandwidth to the equivalent of a single audio stream. After the compressed stream reached the remote site, it was multicast to all participants. We found on numerous occasions that multicasting the audio stream created problems with processor and/or network loads. Some audio packets were either lost or arrived out of order. On these occasions the audio had distinguishable and often unacceptable amounts of "breakup" or short ( 100 millisecond) gaps and noise.

To help reduce the possible processing and network load causing these anomalies, we reconfigured the network layout to use dualhomed hosts at each site, each with a second network to handle the audio stream. This provided some measure of isolation and additional I/O processing power for the audio and collaboration state traffic. Experiments showed this to provide a noticeable improvement in audio quality. Microphone levels and audio feedback caused some echo and distortion on the network, and tuning of the audio for each participant is required. With these issues addressed, the audio quality is sufficient for use of speech recognition and spatialization of speaker audio.

\section{LESSONS LEARNED}

CAVERNSoft provided a useful means for interfacing the HIVE to VisualEyes. Its high-level communication metaphor of "distributed shared memory", indexed by "keys", makes it easy for the HIVE to send commands normally used with 2D interfaces to the 3D VisualEyes client. For example, the avatar's "skull cap" highlights when speaking to provide an additional cue about who is speaking.

The ability to extend a base application such as VisualEyes is becoming common in commercial applications. Any future work on VisualEyes (or commercial versions) should maintain similar extensibility to encourage growth and prevent premature obsolescence.

Our intention was to use Java for HIVE application development. The goal was to use standards to create "write once run anywhere" 
code. Unfortunately, Java classes did not support real-time audio at the time. Therefore, the audio client and server development was written in $\mathrm{C}$ and targeted specifically, via SGI libraries, to SGI machines. Sound effects, spatialization, and voice command programs were restricted to PCs. We have since redeveloped the HIVE to work fully on PCs by extending the Robust Audio Tool (RAT) [5].

To better utilize network bandwidth, the HIVE employs multicasting. Multicasting is a means of making the same information available to anyone on a network who subscribes to it, without replicating the data for each participant. Surprisingly, because of the nature of multicasting, this has at times appeared to be undesirable. Typically multicast traffic on one LAN is isolated or blocked by routers from traversing to other LANs within an intranet. In more elaborate network setups, LANs can be set up explicitly to vary the scope of multicast traffic to a local area, a region, or globally. At HRL our networks are partitioned at each LAN in such a way that multicast traffic does not leave the local departmental area unless specifically designated to do so. At GM $\mathrm{R} \& \mathrm{D}$, we found that the campus network was "flat" relative to multicast traffic. Because of this, all our multicast digital audio traffic was traveling outside the local lab LAN and was available for others on the campus to listen in. While this could be considered a bandwidth-preserving feature if numerous designers wanted to listen in, for our purposes it was using bandwidth unnecessarily. To limit the traffic to the local lab, we set up a newly partitioned network within the lab for multicast traffic. This provided the added benefit of locally dedicated bandwidth.

Audio quality using the HIVE is also difficult to maintain, mainly due to network design constraints and the ambient acoustical environment. On a local area network running fast Ethernet, the HIVE system can convey the full speech spectrum. When all the audio from one site is compressed to the equivalent of one speaker's bandwidth and sent over the limited ISDN, the quality deteriorates. Packets lost or arriving out of order over a congested network can also can cause audio gaps and popping sounds. The use of headphones at workstations provides ideal isolation from audio feedback. However HIVE participants talking in the CAVE causes feedback from nearby speakers that manifests as echoes to remote speakers. The use of new super directional microphone arrays may reduce this undesirable feedback effect in the future. It should also be noted that careful adjustment of the settings on the audio mixing equipment and SGI audio panel controlling microphone and external line out gains are necessary to optimize the audio quality. High quality audio collaboration over widely distributed sites remains a difficult research objective.

Procedurally, the HIVE and VisualEyes setup and runtime research environment is sensitive to process initiation order. There can be a half dozen or so computers at each site, each running several client and/or server processes. Since many of these processes are interdependent, it is necessary to launch them in a particular order or they will either not work as desired or fail to work at all. This is especially true for client server pairs and the ISDN inter-site connection. If a server was not ready for a client, it is possible the client could either fail immediately or repeatedly try to contact the remote server and swamp an ISDN router buffer. This interdependency can result in a time consuming need to restart several pieces of equipment and/or

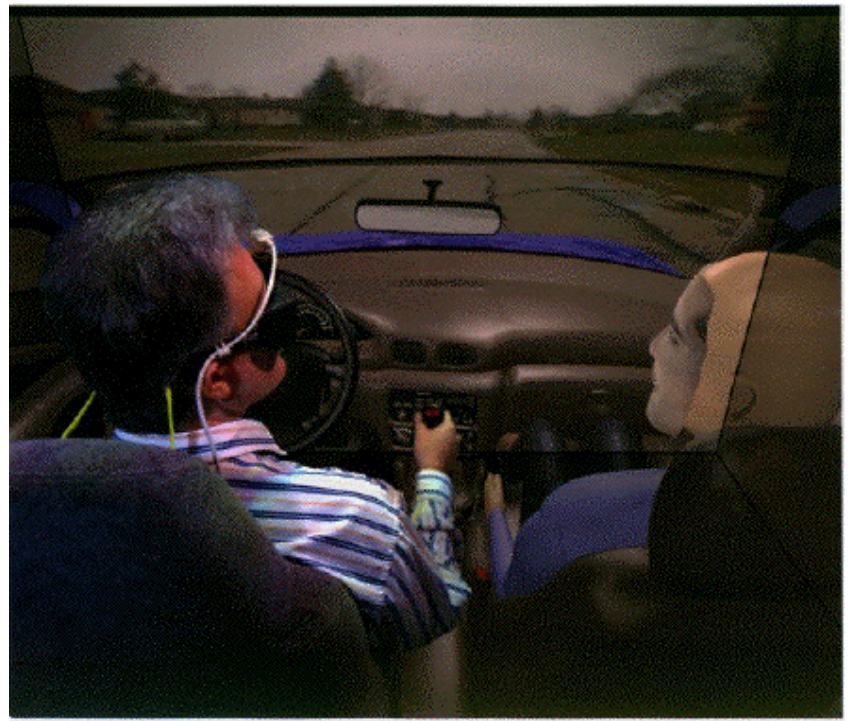

Figure 9. GM R\&D user and HRL avatar with speaker highlighting collaborating inside GM R\&D CAVE.

processes before a demonstration can continue. By documenting the startup procedure, we were able to minimize effort and time.

Command-based spoken interaction in a highly conversational design environment is problematic. Extracting commands, or "word spotting" from continuous speech is difficult. In addition, many of the same words used to command viewpoint navigation and model changes (e.g. forward, rotate, paint, grab) are commonly used in other contexts during the design discussions. Prefixing all speech commands with a pause and a single starting phrase or word (e.g., "Computer, please...") was acceptable to some users but was undesirable and unnatural to others. Methods for canceling or backing out of complex voice command grammars can also be difficult and unnatural from the user interface perspective.

In the VisualEyes environment we made some modest observations. The use of avatars with static faces approximating the actual participants was usually desirable, though some people seemed to prefer generic or non-matching faces for the polar reasons of either vanity or modesty. Filming and videotaping a real and virtual participant in a darkened CAVE is also difficult and requires experienced audio-visual personnel and equipment. Figure 9 is an actual photograph taken in the CAVE at GMR\&D, with enhanced photographic lighting. The real camera location was used for the viewer location to render the images on the CAVE walls.

\section{CONCLUSION}

This paper has described a complex system enabling a widely distributed collaborative virtual environment, as well as a number of lessons learned and technical details of importance in implementing these types of systems. The application of distributed design review in virtual environments motivated the development of key aspects of the system. The DDRIVE system provides an early concept demonstration indicating that it is 
possible to communicate design concepts and ideas with a natural, intuitive interface over great distances. This communication uses visual forms such as avatars, various levels of interaction, spatial audio, and speech recognition, which are key technical elements in the creation of effective collaboration systems.

Development of multi-party, multi-site mixed immersive 3D and 2D collaboration system required several engineering solutions during this effort. A key future goal for polymodal collaboration environments is to support automatic transformation between widely disparate interface modalities rather than the labor intensive approach of engineering specific interface transforms such as speaker highlighting used in this work for both $2 \mathrm{D}$ and $3 \mathrm{D}$ images of users.

\section{ACKNOWLEDGEMENTS}

This project was partially funded by GM R\&D. We gratefully acknowledge the work of the VisualEyes team of Rich Pawlicki, and Larry Peruski at GMR\&D, and Dave Brown at EDS VR Center, and the work of Jason Leigh at UIC EVL for the CAVERNSoft software.

\section{REFERENCES}

[1] Cruz-Neira, C., Sandin, D. J., DeFanti, T. A., Virtual Reality: The Design and Implementation of the CAVE, in Proceedings of SIGGRAPH '93, 1993, 135-142.

[2] Daily, M., Sarfaty, R., Jerald, J., McInnes, D., Tinker, P., The Cabana: A Re-configurable Spatially Immersive Display, in proceedings of the Third International Immersive Projection Technology Workshop, 1999, 123-132.

[3] Glass, G., Voyager, http://www.objectspace.com/products/documentation/Voyag erOverview.pdf

[4] Habanero, http://havefun.ncsa.uiuc.edu/habanero/

[5] Hardman, V. A. Sassie, Kouvelas, Successful Multi-Party Audio Communication over the Internet, Communications of the Association of Computing Machinery, Vol. 41(5), 74-80.

[6] Howard, M., Tinker, P., Martin, K., Lee, C., Daily, M., Clausner, T., T. Lackner, The Human Integrating Virtual Environment (HIVE), in Proc. 13 ${ }^{\text {th }}$ All-Raytheon Software Symposium, 1998.
[7] Jerald, J., Daily, M., Neely, H., Interacting with 2d Applications in Virtual Immersive Environments, Submitted to VRST2000.

[8] Leigh, J., Johnson, A., DeFanti, T., et al., A Review of TeleImmersive Applications in the CAVE Research Network, in Proceedings IEEE VR99, 1999, 180-187.

[9] Leigh, J., Johnson, A., DeFanti, T.A., CAVERN: a Distributed Architecture for Supporting Scalable Persistence and Interoperability in Collaborative Virtual Environments, Journal of Virtual Reality Research, Development and Applications.

[10] Martin, K., Tinker, P., Daily, M., Shared Virtual Reality, HRL Technical Report, 1997.

[11] The MASH Project, University of California, Berkeley, http://mash.cs.berkeley.edu/mash/index.html

[12] Microsoft NetMeeting, http://www.microsoft.com

[13] Oregon Graduate Institute, Center for Spoken Language Understanding, http://clsu.cse.ogi.edu/

[14] Ousterhout, J., Tcl and the Tk Toolkit, Addison-Wesley Publishing Company, 1994.

[15] Placeware, http://www.placeware.com

[16] Smith, R. C., Pawlicki, R. R., Leigh, J., Brown, D, Collaborative VisualEyes, in proceedings of the Fourth International Immersive Projection Technology Workshop, 2000 .

[17] Smith, R. C, Peruski, L., Celusnak, T., McMillan, D.J., Really Getting into Your Work: The Use of Immersive Simulations, in Proceedings of the International Body Engineering Conference (IBEC), Advanced Technologies and Processes, Vol. 25, 1996, and Symposium on Virtual Reality Manufacturing Research and Education, Keynote presentation, Chicago, 1996.

[18] VRCO, CAVELib Users Manual, version 2.7, 1999.

[19] Wenzel, E.M. Virtual Acoustic Displays, Conference on Human Machine Interfaces for Teleoperators and Virtual Environments, 1990.

[20] WorldToolKit, http://www.sense8.com/products/wtk.html 